\title{
Lessons Learned from Non-Pharmaceutical Public Health Responses to Pandemic (H1N1) 2009 in Japan
}

\author{
Koji Wada*
}

Department of Preventive Medicine and Public Health Kitasato University School of Medicine, 1-15-1 Kitasato, Sagamihara, Kanagawa 228-8555, Japan

\begin{abstract}
Public health responses in mitigating influenza pandemics include enhanced surveillance, quarantine, patient management, and social distancing. This manuscript summarizes non-pharmaceutical public health responses in Japan and identifies 10 lessons learned from pandemic (H1N1) 2009.

Pandemic (H1N1) 2009 can be classified into five phases: I) prepandemic; II) overseas, when swine influenza A (H1N1) was identified in Mexico and the United States; III) early localized, when domestic clusters of patients were identified in Japan; IV) smoldering; and V) widespread.

Ten lessons were learned and identified: in the prepandemic phase, 1) the initiatives of high-level decision-making bodies must be enforced effectively and comprehensively, and 2) planning must be flexible and based on a range of scenarios. In the overseas phase, 3) difficulties in decision-making and require the public's trust, 4) human rights must be protected, especially for infected individuals, and 5) efficient response operations are essential because of limited resources. In the early localized phase, 6) acceptance of local governments' initiatives, 7) assumption of the identification of the first domestic patients without overseas travel, 8) strategic public communication for minimizing anxiety, and 9) timely resource allocations with termination of unnecessary responses are necessary. In the widespread phase, 10) criteria for school closures are essential. The implementation of these measures could create greater efficiency in public health responses in the face of another epidemic.
\end{abstract}

Keywords: Pandemic, public health responses, decision-making, lessons learned.

\section{INTRODUCTION}

The emergence of pandemic(H1N1) 2009 provided many countries, including Japan, an opportunity to implement public health responses in preparation for the novel influenza [1, 2]. Generally, the supporting evidence as to the responses' effectiveness against globally emergent diseases is often weak as they are infrequently implemented. In addition, the responses are costly and can have secondary effects [3]. Thus, any lessons learned from this experience should be shared with other countries.

Public health responses are classified as either non-pharmaceutical or pharmaceutical responses. Non-pharmaceutical public health responses for influenza include enhanced surveillance, quarantine, patient management, and social distancing while pharmaceutical public health responses include vaccination and antiviral prophylaxis. Non-pharmaceutical public health responses aim to curb the influx of infected individuals while assessing the time left to prepare the system for outbreaks and to lessen the burden on medical institutions by decreasing the number of newly infected individuals [4].

*Address correspondence to this author at the Department of Preventive Medicine and Public Health Kitasato University School of Medicine, 1-15-1 Kitasato, Sagamihara, Kanagawa 228-8555, Japan; Fax: +81-42-778-9257; Tel: +81-42-778-9352; E-mails: kwada@med.kitasato-u.ac.jp, kwadasgy@umin.ac.jp
While aspects of public health responses in Japan, including those against localized outbreaks, have been reported [57], no paper comprehensively focuses on public health responses to pandemic (H1N1) 2009. This manuscript summarizes non-pharmaceutical public health responses in Japan and identifies 10 lessons learned during pandemic (H1N1) 2009.

\section{TIMELINE FOR PANDEMIC (H1N1) 2009}

Fig. (1) shows the average number of patients per week notified nationally as having pandemic (H1N1) 2009 per sentinel medical institutions in 2009, obtained by weekly national sentinel surveillance [8]. Sentinel surveillance of influenza is an element of the national epidemiological surveillance of infectious diseases. Sentinel medical institutions are required to report weekly on the numbers of outpatients with influenza-like symptoms to the Japanese government through local public health centers [9]. Prefectural governments randomly appoint medical institutions in the sentinel roles and representatively as possible.

The author classified the spread of the disease into five phases: I) prepandemic, II) overseas, III) early localized, IV) smoldering, and V) widespread. Table 1 lists key events chronologically for each phase; dates are given in Japanese Standard Time. The World Health Organization (WHO) first termed the disease swine influenza A (H1N1) but the name was then changed to influenza A (H1N1) on 29 April 2009. From the first of July 2009 it was known as pandemic (H1N1) 2009. This paper also uses this terminology. 


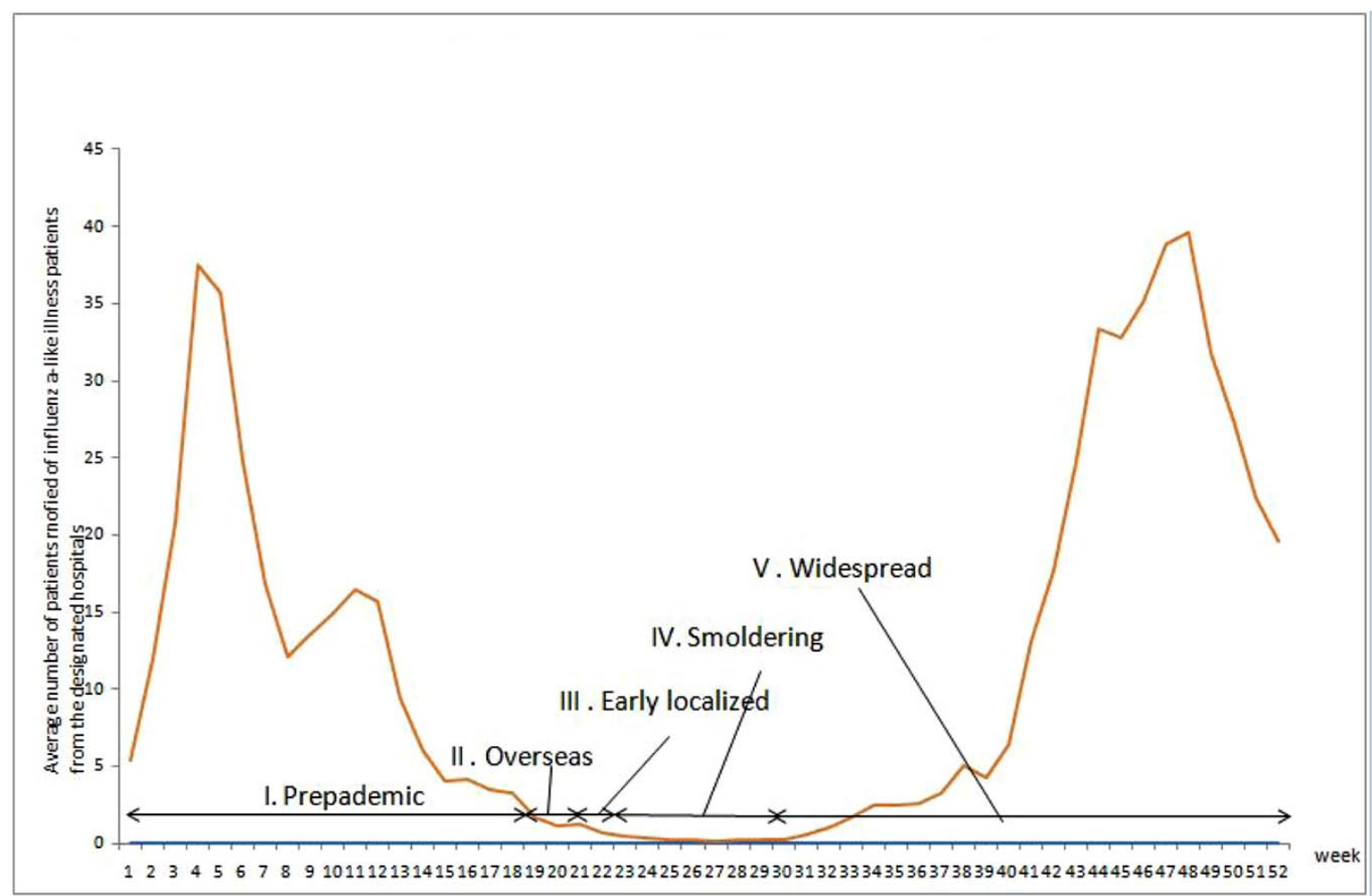

Fig. (1). Average number of notified patients at designated hospitals in 2009 and five phases of pandemic (H1N1) 2009.

Table 1. Key Events for Pandemic (H1N1) 2009 in Japan, 2009

I. Prepandemic phase (before 23 April)

19 February: Published the revised plans of the Japanese Government

II. Overseas phase (from 24 April to 15 May)

24 April: WHO announced that several hundred cases of swine flu in humans in Mexico.

25 April: WHO Director General called public health emergency based on International Health Regulations.

27 April: Declaration of phase 4 by the WHO

28 April: Onboard inspections for passengers from Mexico, the United States and Canada at airports with thermal scanners and health declaration cards

30 April: Declaration of phase 5 by the WHO

8 May: One confirmed case of influenza A(H1N1)2009 from Canada found at Narita International Airport

III. Early localized phase (From 16 May to 31 May)

16 May: Clusters of infection identified in Kobe, Hyogo prefecture, and in Osaka prefecture among junior and high school students

18-24 May: All schools closed in Osaka and Hyogo prefectures

22 May: On board quarantine were cancelled

IV. Smoldering phase (From 1 June to 24 July)

11 June: Declaration of the phase 6 by the WHO

24 July: Stopped to require reports of individual cases of pandemic (H1N1) 2009

V. Widespread phase (From 25 July)

15 August: First case of domestic death due to pandemic (H1N1) 2009 identified in Okinawa

\section{Prepandemic Phase (Before 23 April 2009)}

Japan had not previously been affected by globally emerging infectious diseases. For example, there were no confirmed cases of severe acute respiratory syndrome (SARS) in Japan [10]; therefore, Japan's preparedness for emerging infectious diseases such as novel influenzas was delayed compared with other countries [11].

The National Advisory Committee for Pandemic Flu Preparedness, part of the Ministry of Health, Labour and Welfare (MHLW), had continuously discussed the revision of plans and guidelines for novel influenzas. A national advisory committee rather than a government body published the previous plan and guidelines, which were considered as recommendations only, with the committee having little influence on their implementation.

In 2008, the Japanese public and politicians became aware of the threat of the avian influenza H5N1 pandemic. The cabinet secretariat established a task force and efforts across various ministries and agencies were stepped up; infectious disease controls that had been mainly addressed by the MHLW became matters of crisis management by the Japanese Government. The Act on Infectious Diseases was revised and various public health responses were now legally enforceable during novel influenza outbreaks [12]. In February 2009, the cabinet secretariat published the revised plan and guidelines [2]; resulting in the acceleration of planning measures by Japanese national and local governments.

\section{Overseas Phase (From 24 April to 15 May 2009)}

The swine influenza A (H1N1) epidemic in Mexico was reported by WHO on 24 April 2009, prompting the Japanese Government to begin discussions on the implementation of public health responses [13]. The quarantine office in Narita International Airport began onboard inspections for direct flights from Mexico on 25 April 2009. When WHO declared 
the pandemic at phase 4 on 28 April 2009, the MHLW decided that the swine influenza A (H1N1) virus justified the measures specified in the Act on Infectious Diseases; the implementation of various public health responses. On 28 April 2009, travelers returning from Mexico, the United States, and Canada, which were designated as outbreak regions, were screened; those who had been in contact with infected individuals were detained in quarantine for 10 days.

Public health centers operated fever-consulting hotlines, where the health of travelers returning or coming from overseas was actively monitored and inquiries from local residents about prevention and response measures were answered. There are 510 public health centers in Japan, established in areas with populations greater than 300,000 or with certain geographic characteristics. On 3 May 2009, local laboratories throughout Japan prepared to perform polymerase chain reaction (PCR) testing for influenza $\mathrm{A}$ (H1N1).

Japan had its first confirmed cases of influenza A (H1N1) on 8 May 2009 when four individuals - three high school students and a teacher-were confirmed as infected by PCR assay at the airport on their return from Ontario, Canada [14]. On 15 May 2009, another person who had returned from the United States was also confirmed as infected, but as yet no domestic cases were identified in Japan.

\section{Public Health Responses}

Onboard Inspection at Airports, Quarantine, and Health Monitoring in Communities

Japan is an island nation, meaning overseas visitors can only enter the country via planes or ships. As of 25 April 2009 passengers from Mexico and later the United States and Canada (from 28 April 2009), were required to have their body temperature measured by thermal scanners and to outline their health status on health declaration cards. If any passenger from these countries presented with fever or acute respiratory illness, they were then assessed using a rapid influenza diagnostic kit. If an individual rapid test was positive for influenza $\mathrm{A}$, a PCR assay was performed to confirm the infection was influenza A (H1N1). Once influenza A (H1N1) was confirmed, the patient then received inpatient treatment in isolation in a designated hospital. Individuals who had been seated within two-meters of the patient on board the airplane or those who had traveled with the patient were quarantined for up to 10 days in a hotel secured by the Japanese Government and received antiviral prophylaxis and, in the event of the onset of the disease, treatment.

Onboard inspections were performed on 223,809 travelers from Mexico, the United States, and Canada between 28 April and 22 May 2009; 630 were given rapid influenza diagnostic tests because of suspected fevers as detected by the thermal scanners, the self-declaration of symptoms, or for other reasons. Of the 630 people tested, 8 were found to be positive for influenza $\mathrm{A}$; of these, 4 were confirmed to have influenza A (H1N1) by the PCR assay. However, several people tested positive for influenza A on arrival and returned negative PCR assays for influenza A (H1N1), but were later identified as positive due to the limitations of the rapid kit test.
The 48 individuals who had been seated within twometers of the first confirmed patients of influenza A (H1N1), the three high school students and their teacher, and those who had been in close contact with them, were quarantined at a designated hotel near the airport for 10 days in accordance with The Quarantine Act [14]. However, on 13 May 2009 the length of detention was reduced to 7 days and then all detention measures were terminated on 22 May.

All travelers from Mexico, the United States, and Canada were provided with questionnaires and were required to provide contact details for their time in Japan, to allow for active surveillance. This information was sent to local public health centers, which monitored the travelers' health and any subsequent symptoms over the phone. Between 28 April and 22 May 2009, 117,533 travelers were monitored in this way, but only three people were confirmed as infected with influenza A (H1N1).

\section{Fever Consulting Hotline and Patient Management}

As detailed in the national plan, local public health centers set up a fever consulting hotline. It provided a 24 hour telephone consultation service for individuals with influenza-like symptoms, such as fever, cough, and sore throat. Individuals with influenza-like illnesses were advised to visit a designated medical institution for isolation and treatment and their family members who had had close contact could also be isolated or provided with antiviral prophylaxis for contact management.

To ensure effective infection control, non-designated medical institutions were advised not to see patients with suspected influenza A (H1N1). In addition, if a clinician saw a patient with suspected influenza A (H1N1), he/she was required to report the patient to a local public health center.

\section{Risk Communication}

Information concerning influenza A (H1N1) from countries where the epidemic had already been identified was obtained only through commercial media or informal research networks, thus limiting the information's medical and epidemiological characteristics [15]. There was little confusion before infected patients were confirmed in Japan, but a panic began once suspected cases were identified. As a result, there were some slanderous comments made concerning infected individuals that were seen as a violation of their human rights.

The media and the government provided information on various actions to prevent infection at personal or community levels, such as hand hygiene and respiratory etiquette. Traditionally, hand hygiene, mask wearing, and gargling had been considered standard precautions anyway, and the sales of personal protective equipment, including masks, increased readily. However, the less recognized yet recommended actions for infected individuals, such as voluntary selfisolation and respiratory etiquette, were not promoted.

\section{Early Localized Phase (From 16 to 31 May 2009)}

The first epidemic in Japan was identified in southern central Japan, in the Kansai region on the main island of Honshu [16-18]. On 16 May 2009 several Kobe high school students, who had no history of overseas travel, were 


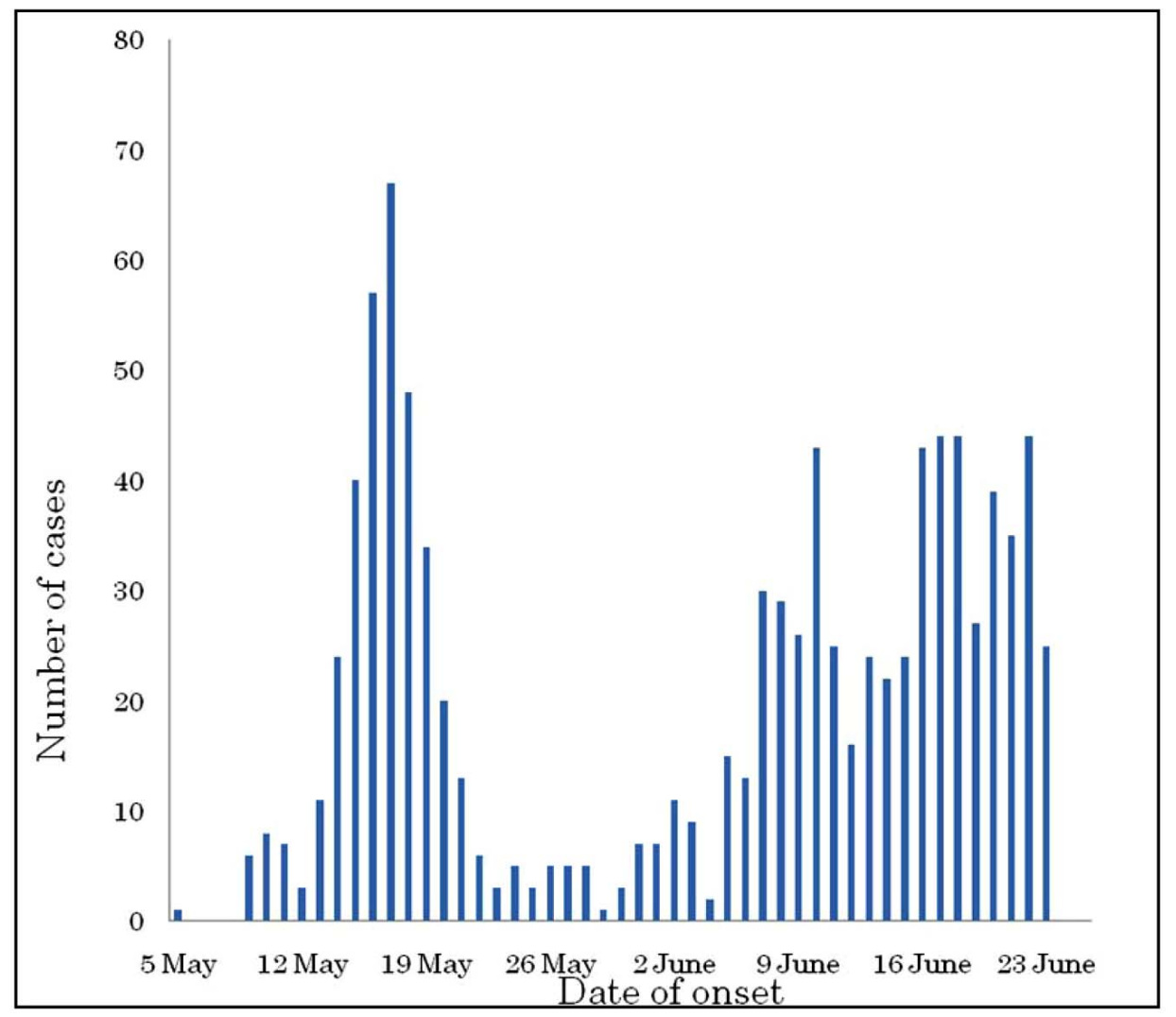

Fig. (2). Notification of laboratory-confirmed cases of Pandemic (H1N1) 2009 infection in Japan by date of onset from 5 May to 23 June.

confirmed as having influenza A (H1N1) by the PCR assay [18]. Samples had been collected earlier on 12 May 2009 by a local clinician but were not tested immediately as the students had not recently been overseas. A further group of infected junior and high school students were also identified in the Osaka Prefecture on 16 May 2009 [18].

Fig. (2) shows the number of notified patients classified by the date of onset, identified by an active epidemiological survey from 5 May to 23 June 2009 [17]. The active investigation suggested that the index case in Kobe, of 5 May 2009, was a person with no history of overseas travel and that the slight community transmission of influenza A (H1N1) had started before then [18].

Containment measures were implemented in the earlylocalized phase: large-scale school closures and the cancellation of mass gatherings were enforced in view of the fact that the infection was spreading among students. Hyogo and Osaka Prefectures promptly closed all schools in the areas (between 18 and 24 May 2009) [18]. After the school closures, reported cases declined sharply, from 30 cases on 17 May to none by 25 May 2009. On 28 May 2009, the Mayor of Kobe declared that the epidemic appeared to be over in the city.

\section{Public Health Responses}

\section{School Closures and Social Distancing}

School closures are divided into two types: proactive closures (closing a school before significant transmission occurs among students and staff) and reactive closure (closing a school when many children and/or staff are ill) [19]. Hyogo and Osaka Prefectures proactively closed all schools from 18 to 24 May 2009. On 18 May 2009, Hyogo Prefecture closed 2,142 schools and Osaka Prefecture closed 1,901, including kindergartens and universities.

The closing of schools and the cancellation of mass gatherings are costly exercises and their scale and timing should be carefully considered [19]. The Kansai region suffered great economic losses as a result of the epidemic, estimated at 121 billion yen (equivalent to US\$ 1.21 billion) [20].

The Mayor of Kobe held a press conference on 28 May 2009 and indicated that the spread of influenza A (H1N1) had apparently been contained and invited visitors into the city. The announcement was issued for political and economic reasons. It is important to protect citizens from infection but an optimal balance between safeguarding the economy and other societal needs must considered in future epidemic planning.

\section{Patient Management and Fever Consulting Hotline}

The action plan specified that during the early localized phase, patients with influenza-like illnesses should first call the fever consulting hotline and patients with suspected influenza A (H1N1) were to be transferred to designated hospitals to control the spread of the disease [2]. However, the fever consulting hotline was completely overwhelmed by the number of calls during this phase. In Hyogo and Osaka Prefectures, the number of phone calls to the fever consulting hotline increased significantly after the infection was confirmed. In Osaka Prefecture, there were 200-300 phone calls per day to the fever consultation hotlines before 
any epidemic was identified in Japan. After the infection was publicly identified on 16 May 2009, there were more than 6,000 phone calls per day for four consecutive days, during which 68 patients in Osaka Prefecture were confirmed to have influenza A (H1N1) [21].

\section{Smoldering Phase (From 1 June to 31 July 2009)}

The rate of influenza $\mathrm{A}(\mathrm{H} 1 \mathrm{~N} 1)$ infections slowed after the school closures in Hyogo and Osaka Prefectures. Mandatory reporting of individual patients to local governments with suspected or confirmed influenza A (H1N1) was discontinued; reporting was only required when an epidemic occurred in certain settings; and inpatient monitoring was required only for critically ill patients (such as those with pneumonia or encephalopathy) and for deaths.

Sporadic outbreaks were identified in many prefectures, but no major epidemic was detected in this phase. This may have been attributable in part to the fact that influenza does not spread so readily during the summer season. However, infected individuals were still identified in all prefectures of Japan. Thus, an epidemic could occur at any time. To best describe this period we have termed it the 'smoldering' phase.

\section{Widespread Phase (From 1 August to 31 December 2009)}

The number of patients with influenza-like illnesses increased after 1 August 2009-a pattern not seen in the previous 10 years. The first widespread outbreak occurred in Okinawa Prefecture, the southernmost area of Japan [22]. Further infected individuals were then confirmed in other parts of Japan, peaking in the week of 23 November 2009. Government sentinel surveillance reported that 17,530,000 individuals (95\% confidence interval: 17,340,000-17,720,000 individuals) were estimated to have been infected with pandemic (H1N1) 2009 by 27 December 2009 [23].

\section{Public Health Responses}

\section{School Closures}

Schools and classes were closed to prevent the spread of infection via passive closure [20]. Class suspension policies were determined by the principal of each school or by local boards of education. School doctors were also meant to have a role in determining these policies but were only consulted on a minor level. The Ministry of Education, Culture, Sports, Science, and Technology reported that class suspensions and school closures among the 52,000 kindergartens, primary schools, junior high schools, and high schools peaked in the week of 26 October 2009 (class suspensions in 14,324 schools; 1,061 school closures) [24].

\section{LESSONS LEARNED}

\section{Prepandemic Phase (Before 23 April 2009)}

Initiatives of High-Level Decision-Making Bodies can Effectively and Comprehensively Enforce Measures Against Emerging Infectious Diseases

The MHLW played a leading role in implementing infection control but other ministries and agencies were also involved in the planning and implementation, as documented in the revised national action plan [2]. Each ministry and agency discussed what they could do: the Ministry of Foreign Affairs focused on measures to protect Japanese living overseas, the Ministry of Defense discussed support for the return of Japanese living overseas, and the Ministry of Education, Culture, Sports, Science, and Technology focused on responses to school closures. The commitment of high-level decision-making bodies can help formulate effective and comprehensive responses.

\section{Plans should be Flexible and Based on Possible Scenarios}

The national plan for pandemic influenza specified a basic policy and assumed worst-case scenarios; it was subject to change as appropriate, in response to new scientific findings or changes in the spread of infection [2]. In developing the plan, the advisory committee discussed the introduction of severity indices for the pathogenicity of the virus, which could include the case fatality rate, excess death rate, illness rate, and the potential number of deaths [25]. While the advisory committee did not introduce the indices, the applicability of severity indices or scenarios merits further study.

\section{Overseas Phase (From 24 April to 15 May 2009)}

\section{Difficulty in Decision-Making with Limited Data and Need for Public Trust}

A government must act quickly in its decision-making to implement public health responses to a novel influenza epidemic; even in the early stages when basic information such as mortality rates and infectivity are poorly sourced (such as from popular media) or lacking entirely [15].

The spread of the Internet and global networking have facilitated information exchange on viral pathogenicity and other relevant issues from outbreak regions. However, the quality and the handling of the information require attention; information can contribute to the strengthening of domestic decision-making but it is not usually sufficient to reduce introduced measures.

Since evidence of the effectiveness of public health responses is often poor, politically based decisions, i.e., those involving the public's trust, are important during this stage [26]. Administrative authorities and experts should continue to build and maintain trust with decision-makers and the public as they would under normal circumstances.

\section{Human Rights of Infected Individuals should not be Violated}

On 1 May 2009 in Yokohama, a high school student who recently returned from Canada developed an influenza-like illness. A rapid influenza diagnostic test showed him to be infected with influenza A, giving rise to suspicion that he may have influenza A (H1N1). In the interest of public information, the Japanese Government held a press conference before influenza A (H1N1) could be confirmed by the PCR assay. The media coverage provided identifiable information, including the name of the school the patient attended, causing great concern to the citizens living in the area until the student was confirmed not to have influenza A (H1N1). 
Responses to the identification of the school that the three high school students and teacher attended (confirmed as infected 8 May 2009) were severe [14]. The school received abusive phone calls, slanderous comments were made on the Internet, there was a refusal to clean the school's uniforms, and other students were ostracized simply because they attended the same school as the patients.

The limited nature and the unreliability of the information available in Japan during the epidemic (this may be related Japan's geographic characteristics as an island nation [27]) may be unacceptable to some people. However, during the SARS outbreak other countries displayed prejudice too, towards Chinese people as the virus originated in China [28]. Such slanderous statements should not be tolerated and should be addressed early in an epidemic by a "rumor control" policy.

\section{Public Health Responses should be Performed Efficiently as Resources are Limited}

Public health responses are labor-intensive, placing a heavy burden on the Quarantine Office, public health centers, and public laboratory centers [3]. The Quarantine Office was required to conduct onboard inspections and to then process any infected individuals. Approximately 220,000 travelers were subjected to onboard inspection; only 4 were confirmed to have the disease at the test site. A further 120,000 travelers returning from Mexico, the United States, and Canada were subjected to health monitoring performed by public health centers. Based on information provided by the Quarantine Office, prior to the completion of health monitoring on 22 May 2009, only three infected patients were identified. Efficient strategies and implementation can help to improve responses when working with limited health care staff.

\section{Early Localized Phase (From 16 to 31 May 2009)}

\section{Allow Local Governments to Take Strategic Initiatives}

The Japanese Government's plan stipulated how to deal with a national epidemic but did not provide sufficient measures at a local level. The ambiguous role of regional authorities in the plan caused some confusion regarding the designation of health care institutions to receive patients. Future plans should indicate that local governments might independently decide some measures, considering the differences in epidemic spread in different regions of Japan.

\section{Assume that Early Stage Epidemics could Involve Patients with no History of Overseas Travel}

It was assumed that the first cases of infection in Japan would be identified through health monitoring among travelers returning from Mexico, the United States, and Canada, where the disease was already confirmed. However, the first infected patients were identified in Kobe and had no history of overseas travel. For this reason, their PCR influenza A (H1N1) assays were given low priority, thus delaying diagnosis for several days. If a novel influenza has been identified in other countries, it should be assumed that transmission might emerge in Japan among individuals with no history of overseas travel.

\section{Strategic Communication is required to Allay Public Anxiety}

Public anxiety peaked when the first cases of infection were detected in Japan, as shown by the increasing number of calls to fever consultation centers. In such situations, the burden on fever consulting hotlines and medical institutions should be reduced through timely communication with the public.

The media reported on actions for the prevention of infection but there was little information on measures appropriate to the risk of infection. Influenza is generally transmitted by respiratory droplets from coughs and sneezes and there is little possibility of infection if there are no infected individuals present. An overreaction then occurred when expert medical support was not available to help implement the appropriate infection control measures.

\section{Start and Termination of Public Health Actions should be Timed for Optimal Allocation of Limited Resources}

Public health responses are labor-intensive and costly, and impact significantly on society [3]. It should be made clear to decision-makers early in an epidemic that human and other resources are limited and must be optimally allocated. Criteria for terminating or changing actions and strategies should be defined before their initiation; alternatively, regular weekly meetings could be scheduled to discuss these issues. The termination of unnecessary public health responses will produce some adverse effects but all the while, the best possible allocation of resources should remain the overall aim.

\section{Widespread Phase (From 1 August to 31 December 2009)}

\section{Criteria for School Closures should be Established and Clearly Communicated to Students and Parents}

An investigation by the Ministry of Education, Culture, Sports, Science, and Technology revealed that by the end of August 2009, only 15 of the 47 prefectures in Japan met the criteria for school closure. Many of the class suspensions implemented during this period were reactive school closures and had limited effect. The criteria for class suspension requires further refinement, with decision-making systems that are aided by professionals and are acceptable to both parents and the community.

\section{NECESSITY OF REVISED PLANNING AND FUR- THER PREPARATION}

We should use the lessons learned from this experience in considering optimal public health responses in preparation for future outbreaks of novel influenzas or other emerging diseases. The swine influenza A (H1N1) 2009 was identified in Mexico by WHO on 24 April and 12 days later the first infected individuals in Japan were identified at an airport on 8 May 2009. Eight days later domestic community transmission was confirmed. While Japan had some time to prepare its public health responses, it is not possible to know how much preparation time there will be for any future outbreaks. Therefore, the Japanese Government and health 
practitioners must continue to improve the national epidemic plan, and similar plans, using the lessons learned from pandemic (H1N1) 2009.

\section{REFERENCES}

[1] Novel Swine-Origin Influenza A(H1N1) Virus Investigation Team, Dawood FS, Jain S, Finelli L, et al. Emergence of a novel swineorigin influenza A (H1N1) virus in humans. N Engl J Med 2009; 360: $2605-15$.

[2] Ministry of Health, Labour and Welfare, Japan. Pandemic influenza and avian influenza. [Online]. 2010 Apr 13, Available from: http://www.mhlw.go.jp/english/topics/influenza/index.html

[3] Aledort JE, Lurie N, Wasserman J, Bozzette SA. Non-pharmaceutical public health interventions for pandemic influenza: an evaluation of the evidence base. BMC Public Health 2007; 7: 208.

[4] European Centre for Disease Prevention and Control. Guide to public health measures to reduce the impact of influenza pandemics in Europe. 2009. [Online]. 2010 Apr 13, Available from: http://www.ecdc.europa.eu/en/publications/Publications/0906_TER _Public_Health_Measures_for_Influenza_Pandemics.pdf

[5] Yasuda ${ }^{H}$, Suzuki K. Measures against transmission of pandemic H1N1 influenza in Japan in 2009: simulation model. Euro Surveill 2009; 14: pii 19385

[6] Tsubokura M, Nakada H, Matsumura T, Kodama Y, Kami M. The impact of H1N1 influenza A virus pandemic on the emergency medical service in Kobe. Am J Emerg Med 2010; 28: 248-51.

[7] Sato H, Nakada H, Yamaguchi R, Imoto S, Miyano S, Kami M. When should we intervene to control the 2009 influenza A (H1N1) pandemic? Euro Surveill 2010; 15: pii 19455.

[8] Infectious disease surveillance center, Japan. Influenza cases reported per sentinel weekly. [Online]. $2010 \mathrm{Apr} 13$, Available from: http://idsc.nih.go.jp/idwr/kanja/weeklygraph/01 flu-e.html

[9] Hashimoto S, Murakami Y, Taniguchi K, et al. Annual incidence rate of infectious diseases estimated from sentinel surveillance data in Japan. J Epidemiol 2003; 13:136-41.

[10] World Health Organization. Severe acute respiratory syndrome [Online]. 2010 April 13, Available from: http://www.who.int/csr/ sars/en/

[11] Coker R, Mounier-Jack S. Pandemic influenza preparedness in the Asia-Pacific region. Lancet 2006; 368: 886-9.

[12] Act on Prevention of Infectious Diseases and Medical Care for Patients Suffering Infectious Diseases. (in Japanese) [Online]. 2010 Apr 13, Available from: http://law.e-gov.go.jp/htmldata/H10/ H10HO114.html

[13] WHO. Influenza-like illness in the United States and Mexico. [Online]. 2010 Apr 13, Available from: http://www.who.int/csr/ don/2009_04_24/en/index.html

[14] Narita International Airport Quarantine. A report of response for pandemic (H1N1)2009 in the Narita International Airport.22th July 2009 (in Japanese) [Online]. 2010 Apr 13, Available from:
http://www.forth.go.jp/keneki/narita/pdf/20090805_NQ_H1N1\%20 flu Report.pdf

[15] Lipsitch M, Riley S, Cauchemez S, Ghani AC, Ferguson NM. Managing and reducing uncertainty in an emerging influenza pandemic.N Engl J Med 2009; 361: 112-5.

[16] National Institute of Infectious Diseases, Osaka Prefecture and Public Health Center of Osaka Prefecture. Interim report on two clusters of the novel influenza A (H1N1) infection in Osaka Prefecture. [Online]. 2010 Apr 13, Available from: http://idsc.nih. go.jp/disease/swine_influenza_e/idsc_e2009/clinical_epi_osaka2.ht $\mathrm{ml}$

[17] National Institute of Infectious Diseases. Number of cases by date of onset. [Online]. 2010 Apr 13, Available from: http://idsc.nih. go.jp/disease/swine influenza/epi2009/090710epi.html

[18] Shimada T, Gu Y, Kamiya H, et al. Epidemiology of influenza A (H1N1)v virus infection in Japan. May-June 2009. Euro Surveill 2009; 14: pii19244.

[19] Cauchemez S, Ferguson NM, Wachtel C, et al. Closure of schools during an influenza pandemic. Lancet Infect Dis 2009; 9: 473-81.

[20] Kansai Institute for Social and Economic Research. Report on the effect of pandemic flu in Kansai, October 2009. [Online]. 2010 Apr 13, Available from: http://www.kiser.or.jp/ja/temp/pdf/826_Pdf02. pdf

[21] Osaka Prefecture.Responses for pandemic A(H1N1)2009 in Osaka. (in Japanese) [Online]. 2010 Apr 13, Available from: http://www. pref.osaka.jp/attach/6188/00027599/hudou0814.pdf

[22] Shimada T, Toyokawa K, Sunagawa T, Taniguchi K, Okabe N. Epidemiological survey of pandemic (H1N1) 2009 in Okinawaprefecture (in Japanese) [Online]. 2010 Apr 13, Available from: http://idsc.nih.go.jp/disease/swine_influenza/pdf09/Okinawa_0914 09.pdf

[23] Ministry of Health, Labour and Welfare. Report on sentinal surveillance for influenza (in Japanese) [Online]. 2010 Apr 13, Available from: http://www.mhlw.go.jp/kinkyu/kenkou/influenza/ houdou/2010/01/dl/infuh0115-02.pdf

[24] Ministry of Education, Culture, Sports, Science and Technology. The number of school closures and class suspensions. (in Japanese) [Online]. $2010 \mathrm{Apr}$ 13, Available from: http://www.mext.go.jp/ a_menu/influtaisaku/syousai/_icsFiles/afieldfile/2010/03/19/1266 888_2.pdf

[25] Centers for disease control and prevention. Community strategy for pandemic influenza mitigation. 2007. [Online]. 2010 Apr 13, Available from: http://www.flu.gov/professional/community/ commitigation.html

[26] Larson HJ, Heymann DL. Public health response to influenza A (H1N1) as an opportunity to build public trust. JAMA 2010; 303: 271-2.

[27] Shigemura J, Nakamoto K, Ursano RJ. Responses to the oubreak of novel influenza A (H1N1) in Japan: risk communication and shimaguni konjo. Am J Disaster Med 2009; 4: 133-4.

[28] Eichelberger L. SARS and New York's Chinatown: the politics of risk and blame during an epidemic of fear. Soc Sci Med 2007; 65: 1284-95.

(C) Koji Wada; Licensee Bentham Open.

This is an open access article licensed under the terms of the Creative Commons Attribution Non-Commercial License (http://creativecommons.org/licenses/by$\mathrm{nc} / 3.0 /$ ), which permits unrestricted, non-commercial use, distribution and reproduction in any medium, provided the work is properly cited. 